\title{
„Daß es ein Wahnsinn ist, wie wir zur Zeit die Welt bewirtschaften" Herbert Gruhl und Ein Planet wird geplündert
}

\section{Torsten Kathke}

Dass Baden-Württembergs grüner Ministerpräsident Winfried Kretschmann 2016 die erste grün-schwarze Koalition auf Landesebene anführt, erscheint angesichts der verbreiteten Sichtweise, die Grünen fänden sich im politischen Spektrum gänzlich links der SPD wieder, bemerkenswert. Trotz mehrerer schwarz-grüner Koalitionen auf Landes- und Kommunalebene haftet der Verbindung von Grünen und Christdemokraten noch immer etwas Befremdliches an. Sowohl Mitglieder der beiden Koalitionsparteien als auch Kommentatoren in den Medien sahen die neue politische Zweckehe skeptisch. ${ }^{1}$

Für Kretschmann selbst, einst Sprecher der konservativen Ökolibertären unter den Grünen, dürfte die Koalition mit der CDU allerdings keine große weltanschauliche Anstrengung bedeutet haben. ${ }^{2}$ Man mag das mit einer Verschiebung im politischen Koordinatensystem begründen, gar darüber spekulieren, ob zwei Große Koalitionen innerhalb der letzten zehn Jahre den Unterschied zwischen den beiden großen Volksparteien verwischt haben. ${ }^{3}$

Das aber würde die historische Genese der Grünen ignorieren, und speziell die Bedeutung des konservativ-naturkonservatorischen Flügels. Nicht nur in Baden-Württemberg waren Ende der 1970er Jahre Ökologie und Konservatismus vereinbar. Wie Herbert Gruhl, bis 1978 Mitglied des Deutschen Bundestages in der CDU-Fraktion, zwei Jahre später dann Grünen-Gründungsmitglied

1 Jan Friedmann: Grün-Schwarz in Baden-Württemberg. „Wir haben uns nicht gesucht, aber wir haben uns gefunden“, in: Spiegel Online, 2. Mai 2016 (http://www.spiegel.de/politik/deutschland/gruen-schwarz-in-baden-wuerttemberg-gruene-und-cdu-besiegeln-koalition-a-

1090459.html, Abruf: 27. Mai 2016); Josef Kelnberger: Grün-Schwarz in Baden-Württemberg. Partner wider Willen, in: Süddeutsche Zeitung, 2. Mai 2016; allgemein zu schwarz-grünen Koalition: Volker Kronenberg/Christoph Weckenbrock (Hg.): Schwarz-Grün. Die Debatte. Wiesbaden 2011.

2 Der Grünen-Politiker und Verfasser einer "subjektiven Chronik” der Partei, Ludger Volmer, weist darauf hin das Label „Ökoliberale“ sei passender. Ludger Volmer: Die Grünen. Von der Protestbewegung zur etablierten Partei. Eine Bilanz. München 2009, S. 167.

3 Ein Vorwurf der seit Otto Kirchheimers Definition der „Catch-All-Party“ oder „Allerweltspartei“ so oft vorgebracht wie bestritten wird. Otto Kirchheimer: Der Wandel des westdeutschen Parteisystems, in: Politische Vierteljahresschrift 6 (1965), S. 20-41; zur Widerlegung z. B Uwe Wagschal: Der Wahl-O-Mat und die politischen Inhalte. Warum sich Union und SPD immer noch unterscheiden, in: Tagesspiegel Online, 16. September 2013 (http://www.tagesspiegel.de/meinung/der-wahl-o-mat-und-die-politischen-inhalte-warum-union-und-spd-sichimmer-noch-unterscheiden/8800372.html, Abruf: 27. Mai 2016). 
(und damit auf gewisse Weise der erste Grüne im Bundestag), nicht müde wurde zu betonen, seien die Grünen die „wahren Konservativen“. ${ }^{4}$

Gruhl hatte mit seinem 1975 veröffentlichten Bestseller Ein Planet wird geplündert ein Kult- und Schlüsseldokument der grünen Bewegung geschrieben. Wie Erhard Eppler, Mitte der 1970er Jahre Gruhls Gegenpart bei der SPD was Umweltmahnungen betraf, schon 1983 anerkennen musste: „Wer auch immer die Geschichte der Ökologiebewegung schreiben sollte, wird um den Namen Gruhl nicht herumkommen. Kein Buch eines aktiven Politikers seit dem Zweiten Weltkrieg hat so eingeschlagen wie Herbert Gruhls ,Ein Planet wird geplündert'."

Eppler ging sogar noch weiter und verband die in den 1980ern stark angestiegene Bedeutung des Umweltthemas in der deutschen Politik direkt mit der Wirkung von Gruhls Buch: „1975 erschienen, hat es nicht nur über Jahre die Bestsellerlisten geziert, es hat auch die Ökologiediskussion - bis dahin als Luxus-Spleen gelangweilter Mittelständler abgetan - zu einem Thema gemacht, an dem sich niemand mehr vorbeidrücken konnte $[\ldots]^{“ .5} .5$

Das Buch war sichtlich beeinflusst von der ab 1972 breit rezipierten Studie Die Grenzen des Wachstums für den Club of Rome. Es schloss an Traditionen sowohl der Kulturkritik, als auch der Wissenspopularisierung und der SelfHelp-Literatur an. In einer Zeit, in der politische Mobilisierung, Bildungsexpansion und Innovationen im Buchmarkt im Kontext der westdeutschen Konsumgesellschaft der Nachkriegszeit zu einer Schwemme von Sachbuchliteratur geführt hatten, geriet der Verweis auf Gruhls Buch geradezu zum Schibboleth für die mannigfaltigen gesellschaftlichen Bewegungen, die zukünftig zur Grünen Partei gerinnen sollten. Und dies obwohl, wie Silke Mende bemerkt, „Gruhls Werk über weite Strecken ein kulturkritisches Manifest mit rückwärtsgewandten, teilweise autoritären Staats- und Gesellschaftsvorstellungen war". Das aber schien zumindest in den Bestsellerjahren des Buchs in der Rezeption weitgehend ausgeblendet zu sein. ${ }^{6}$

Herbert Gruhls Ein Planet wird geplündert, so meine übergeordnete These, war sowohl singuläres Medienprodukt, das nur in einem bestimmten historischen Moment eine derart immense Popularität erreichen konnte, als auch ein Spiegel der Medienlandschaft in der Bundesrepublik Deutschland der 1970er

4 Silke Mende: „Nicht rechts, nicht links, sondern vorn“. Eine Geschichte der Gründungsgrünen. München 2011, S. 92. Zu zeitgenössischen Verschiebungen im Konservatismusbegriff siehe ebd., S. 419-427. Mendes Buchtitel bezieht sich auf einen Slogan der frühen Grünen, die sich im politischen Spektrum ,nicht rechts, nicht links, sondern vorn“ verorten wollten. Dieser wird oft Herbert Gruhl zugeschrieben.

5 Beide Zitate: Erhard Eppler: Vom Fall in das politische Nichts, in: Der Spiegel, 18. April 1983, S. 86f., hier S. 86.

6 Mende: „Nicht rechts, nicht links“, S. 76. 
Jahre. Indem das Buch eine zwar in vielerlei Hinsicht schon öffentlich diskutierte Problematik der Umweltzerstörung und Wachstumskritik auf populäre, gegenwartsdiagnostische Weise engführte, konnte es zum Bestseller aufsteigen und durch die mit dieser Verbreitung gewonnene Autorität politische und gesellschaftliche Handlungen direkt wie indirekt beeinflussen. ${ }^{7}$ Dies geschah in einem durch spezifische Verlags- und Werbestrategien sowie Konsumweisen für Literatur im weiteren, und Sachliteratur im engeren Sinne geprägten Medienkontext.

Durch die herausragende Rolle von Ein Planet wird geplündert stellt sich die Frage nach der Bedeutung dieser Art Literatur allgemein für die Meinungsbildung in der Bundesrepublik der 1970er und 1980er Jahre. Dazu sind verschiedene Betrachtungsdimensionen notwendig. Ich beginne mit einer allgemeinen Einordnung, an die sich eine Übersicht relevanter Forschungsliteratur anschließt. Der folgende empirisch-analytische Hauptteil gliedert sich in drei Unterpunkte, die von der Person über das Werk in die Gesellschaft ausgreifen. Ein Ausblick auf Gruhls Spätwerk und sein politisches Schicksal in den 1990er Jahren schließt die Darstellung ab.

\section{Einordnung}

Gruhl steht zum einen im Kontext konservativer Kulturkritik und Gegenwartsdiagnostik des 20. Jahrhunderts. Zum anderen steht er in Zusammenhang mit Autorenbildern und Konstruktionen des Sachbuchautors und „public intellectual“ in der Bundesrepublik der Zeit „,nach dem Boom“ und dem Medien- und

7 In diesem Aufsatz werden die Begriffe Gegenwarts-, Zeit- und Gesellschaftsdiagnose weitgehend synonym benutzt. Auch wenn die Begriffe mitunter verschiedene Konnotationen hervorrufen, so gibt es keine klare, verbreitete Typologie dieser Unterschiede. Tendenziell ist Zeitdiagnose dabei der umfassendste Begriff, während Gegenwarts- und Gesellschaftsdiagnose temporal (eine wie auch immer geartete „Gegenwart“) oder räumlich (z. B. die ,westliche Gesellschaft“ oder „deutsche Gesellschaft“) einschränken. Der Begriff der Zivilisations- oder Kulturkritik liegt dabei quer zu den drei anderen Begriffen und kann selbst verschieden weit oder eng gefasst werden. Da Gruhls Buch sich hauptsächlich auf Problematiken eines spezifischen, gegenwärtigen, als krisenhaft empfundenen Moments bezieht, der aber sowohl in den im Buch angesprochenen Referenzräumen als auch in den notwendigen Maßnahmen letztendlich globa zu denken ist, habe ich mich zur Bezeichnung Gegenwartsdiagnose entschieden sofern ich den Begriff nicht aus Quellen oder Sekundärliteratur übernehme. Zur Problematik generell siehe: Georg Bollenbeck: Eine Geschichte der Kulturkritik. Von Rousseau bis Günther Anders. München 2007; Ralf Konersmann: Kulturkritik. Frankfurt/Main 2008; Ole Petras/Kai Sina (Hg.) Kulturen der Kritik. Mediale Gegenwartsbeschreibungen zwischen Pop und Protest. Dresden 2011; Uwe Schimank/Ute Volkmann (Hg.): Soziologische Gegenwartsdiagnosen I. Eine Bestandsaufnahme. 2. Auflage Wiesbaden 2007; Fran Osrecki: Die Diagnosegesellschaft. Zeitdiagnostik zwischen Soziologie und medialer Popularität. Bielefeld 2011. 
Öffentlichkeitsraum in dem diese entstanden waren und sich weiterentwickelten. ${ }^{8}$ Dabei bietet die Betrachtung des Buches mikrohistorischen Zugang zum besseren Verständnis eines gesellschaftlichen Resonanz- und öffentlichen Diskursraumes, in dem in den 1970er und 1980er Jahren wichtige Fragen und Ideen verhandelt, verändert und daraufhin in politische Handlungen umgesetzt werden konnten.

Wie konnte ein Buch wie Ein Planet wird geplündert gerade in dieser Ära derart populär werden? Wie konnte Gruhls Vision an andere gesamtgesellschaftliche Debatten einerseits, wie auch spezifische in diesem historischen Moment verortete Diskursformen andererseits anknüpfen? Wie gestaltete sich die öffentliche Reaktion auf Gruhls Thesen, und wie wurden diese sowohl von den Medien als auch den Lesern des Buches reflektiert, übernommen und transformiert? Welche Vorbilder hatte Gruhls Werk, welche rhetorischen und narrativen Strategien verfolgte es, und inwiefern trugen diese zu seinem Erfolg und seiner Anbindungsfähigkeit an Diskurse verschiedenster Bevölkerungsgruppen bei?

Neben der Auswertung von medialer Berichterstattung über Gruhls Ein Planet wird geplündert stützt sich der Aufsatz auf im Archiv für Christlich-Demokratische Politik gesichtete Quellen zu Gruhls Parteiarbeit in der CDU, zu seinem Engagement in der Umweltpolitik, sowie auf Korrespondenz und Selbstzeugnisse Gruhls.

\section{Literaturübersicht und Anschluss an die Forschung}

Gruhls Bestseller Ein Planet wird geplündert. Die Schreckensbilanz unserer Politik verkaufte sich ab 1975400.000 mal. ${ }^{9}$ Die Grünen sind inzwischen die

8 So die gängig-griffige Formel von Anselm Doering-Manteuffel und Lutz Raphael für die Zeit nach 1970. Lutz Raphael/Anselm Doering-Manteuffel: Nach dem Boom. Perspektiven auf die Zeitgeschichte seit 1970. 3. Auflage Göttingen 2012. Das „Sachbuch“ war zwar nicht thematisch, aber doch etymologisch eine Geburt der Nachkriegszeit. Das erste Mal tauchte der Begriff im Duden 1963, ein Jahr nach Einführung der in Sachbuch und Belletristik gegliederten Bestsellerlisten des „Spiegel“, auf. Klaus Ziermann: Der deutsche Buch- und Taschenbuchmarkt, 1945-1995. Berlin 2000, S. 87, 89.

9 Sachbuchforschung.de. Datenbank zur Sachbuchforschung. http://www2.hu-berlin.de/sachbuchforschung/CONTENT/SBDB/SBDB.php5?anzeige=true\&ID=361 (Abruf: 28. Mai 2016). Allein die Erst- sowie die Taschenbuchausgabe des S. Fischer Verlags belaufen sich auf über 300.000 Bücher. Dazu kommt noch eine in hoher Zahl abgesetzte Bertelsmann Buchclubausgabe. Bärbel Höhn sprach 2009 im Deutschlandfunk von insgesamt 400.000 verkauften Exemplaren. Bärbel Höhn: Kursiv. Ein echter Öko-Bestseller. Deutschlandfunk, 7. Dezember 2009 (http://www.deutschlandfunk.de/kursiv-ein-echter-oeko-bestseller.1310.de.html?dram:article_id=193988, Abruf: 2. Juni 2016). 
bestuntersuchte Partei der Bundesrepublik. ${ }^{10}$ Für viele ihrer Gründungsmitglieder war das Lesen von Gruhls Buch ein auslösendes Moment der Politisierung und Aktivierung. ${ }^{11}$ Eine tiefergehende Betrachtung Herbert Gruhls als Autor auf der einen, und des populären gesellschaftsdiagnostischen Sachbuchs als äuBerst bedeutendem Medienphänomen der 1970er und 1980er Jahre auf der anderen, steht jedoch noch weitgehend aus. Volker Kempfs Herbert Gruhl. Pionier der Umweltsoziologie leistet selbst Pionierarbeit in der biographischen Beschreibung Gruhls, bindet sein Buch aber nicht umfassend in die Medienlandschaft der Zeit ein. Silke Mende (,Nicht rechts, nicht links, sondern vorn “) und Oliver Geden (Rechte Ökologie) beschäftigen sich eingehend mit Gruhls Einfluss auf verschiedene Kräfte in den Neuen Sozialen Bewegungen und den sich gründenden Grünen. ${ }^{12}$ Jürgen Wüsts Untersuchung Konservatismus und Ökologiebewegung geht auf Gruhls Rolle in der Gründung der ÖDP ein und setzt sowohl diese als auch die Grünen in einen umfassenden ideengeschichtlichen Zusammenhang. Allerdings beschreibt auch Wüst nicht spezifisch Gruhls Rolle als populärer Autor. ${ }^{13}$

Volker Kruses Historisch-soziologische Zeitdiagnosen in Westdeutschland nach 1945 konzentriert sich zwar in angemessener Tiefe auf nur drei Autoren - Eduard Heimann, Alfred von Martin und Hans Freyer -, endet aber 1965. Kruse zufolge ist dies für soziologische Zeitdiagnosen symptomatisch, da sich die westdeutsche Soziologie nach dem Zweiten Weltrieg vom „Weimarer“ Modell in der Tradition Werner Sombarts und Max Webers verabschiedet und ihr „mainstream“ sich nun unter Federführung René Königs und Helmut Schelskys

10 Wenn auch hauptsächlich aus sozial- und politikwissenschaftlicher Sicht, und weniger aus historischer. Edgar Wolfrum: Die geglückte Demokratie. Geschichte der Bundesrepublik Deutschland von ihren Anfängen bis zur Gegenwart. Bonn 2007, S. 407. Neuere historische Forschung hat sich dieses Defizits allerdings inzwischen angenommen. So etwa Mende: „Nich rechts, nicht links“ und Saskia Richter: Die Aktivistin. Das Leben der Petra Kelly. München 2010.

11 So etwa für Maximilian Gege. Camilla John: Der Manager unter den Naturschützern, in: Hamburger Abendblatt, 3. September 2011

12 Kempf ist Vorsitzender der Herbert-Gruhl-Gesellschaft e. V. die sich laut Satzung ,um die Pflege, Förderung und Weiterentwicklung seines Werkes" kümmert und Anlaufstelle sein will für „Menschen, die sich ihm weltanschaulich verbunden fühlen“. Satzung der Herbert-GruhlGesellschaft. http://herbert-gruhl.de/satzung/ (Abruf: 28. Mai 2016). Volker Kempf: Herbert Gruhl. Pionier der Umweltsoziologie. Graz 2008; Mende: „Nicht rechts, nicht links“; Oliver Geden: Rechte Ökologie. Umweltschutz zwischen Emanzipation und Faschismus. Berlin 1996.

13 Jürgen Wüst: Konservatismus und Ökologiebewegung. Eine Untersuchung im Spannungsfeld von Partei, Bewegung und Ideologie am Beispiel der Ökologisch-Demokratischen Partei (ÖDP). Frankfurt 1993. 
mehr und mehr dem US-amerikanischen Modell einer quantifizierend-positivistischen Wissenschaftlichkeit zugewendet hätte. ${ }^{15}$ Fran Osrecki (Die Diagnosegesellschaft) schließt an Kruses Überlegungen an, präsentiert jedoch ein anders akzentuiertes Bild: nicht allein ein ,inhaltlich unbegründete[r] Kontinuitätsbruch“, sondern eine Ausdifferenzierung der Publika der Soziologie in akademische und nicht-akademische Teile habe zum Niedergang des Wissenschaftsparadigmas Zeitdiagnostik geführt, nie jedoch zum Niedergang der Zeitdiagnostik selbst. ${ }^{16}$

Indirekt weisen Kruse und Osrecki damit auf eine „Marktlücke“ für Zeitdiagnosen nicht-soziologischer Art ab den späten 1960er Jahren hin, in die Bücher wie Gruhls Planet stoßen konnten. ${ }^{17}$ Ebenso leitet sich daraus auch eine Lücke in der Forschung ab: soziologische Literatur, die sich mit Zeitdiagnosen beschäftigt, konnte einen Großteil der zeit- und gegenwartsdiagnostischen Literatur in der Zeit ab 1965 nicht greifen, da diese Literatur zwar zeitdiagnostisch, aber nicht soziologisch war. Ebenso indirekt auf eine Lücke in dieser Forschung verweist der von Carsten Kretschmann herausgegebene Sammelband Wissenspopularisierung. Konzepte der Wissensverbreitung im Wandel. Kretschmann und die Autoren des Bandes beschäftigen sich mit Wissenspopularisierung in all ihren Spielarten von der Antike bis ins späte 20. Jahrhundert, jedoch nicht mit dem Themenkomplex der populären Gegenwartsdiagnose. ${ }^{18}$ Das Buch als eine der bekanntesten der in den 1970er Jahren, der Hochzeit des po-

15 Volker Kruse: Historisch-soziologische Zeitdiagnosen in Westdeutschland nach 1945. Eduard Heimann, Alfred von Martin, Hans Freyer. Frankfurt 1994, S. 9-17, 18-24, 42-49, insbes. S. 13-15.

16 Osrecki: Diagnosegesellschaft, S. 17, 71.

17 Volker Kempf requiriert Gruhl dann auch - gleich im Titel seines Buches, und dann weiter darauf eingehend - für die Soziologie in der Form einer von Gruhl begründeten Umweltsoziologie. Der Zeitdiagnose-Begriff ist stark mit der aus der soziologischen Fachwissenschaft heraustretenden soziologischen Zeitdiagnose verbunden (vgl. Osrecki: Diagnosegesellschaft, passim; Ute Volkmann: Soziologische Zeitdiagnostik. Eine wissenssoziologische Ortsbestimmung, in: Soziologie 44 (2015), S. 139-152, hier S. 139). Kempfs Gedankengang ist damit zwar nach Osreckis Feststellung, Zeitdiagnosen haben sich zwar als Art des „doing science“ in der Soziologie überlebt, seien aber gewisserweise deren medialer Arm, bestechend. Ich setze jedoch einen engeren Begriff soziologischer Zeitdiagnosen an. Da Gruhls Buch nicht als Beitrag zur soziologischen Fachwissenschaft gemeint, und auch in der Fachsoziologie nicht als spezifisch zu dieser gehörend reflektiert wurde, erfüllt es das Kriterium der spezifisch soziologischen Zeitdiagnose nicht

Schließlich weist auch die einschlägige Internetressourcensammlung sachbuchforschung.de über den Publikationshinweis in der Datenbank hinaus keinen Artikel oder Eintrag zu Gruhls Ein Planet wird geplündert auf. http://www.sachbuchforschung.de (Abruf: 28. Mai 2016).20 Siehe hierzu: Gérard Genette: Paratext. Das Buch vom Beiwerk des Buches. Frankfurt 2001. Zur Fruchtbarmachung Genettes für nicht-narrative Literatur in der geschichtlichen Betrachtung siehe: Anna Karla: Revolution als Zeitgeschichte. Memoiren der Französischen Revolution in der Restaurationszeit. Göttingen 2014. S. 116. 
pulären Sachbuchs, veröffentlichten Diagnosen, dient dabei erstens zur allgemeinen Darstellung des Wirkungszusammenhangs, in dem das Buch Bedeutung entfalten konnte und in einem zweiten Schritt zur Feststellung der besonderen Umstände, die gerade dieses Buch so einflussreich werden ließen.

Dazu muss man in zwei Richtungen ausgreifen: zum einen in das Buch selbst. $^{20}$

Zum anderen müssen die Zusammenhänge, in denen das Buch erschien, betrachtet werden.. Zuvorderst ist hier der Umwelt- und Wachstumsdiskurs, dersich in den 1970er Jahren entspann, zu analysieren. Die in der Öffentlichkeit diskutierten Themen beeinflussten stets das Interesse an bestimmten Deutungsangeboten. Andersherum galt aber auch, dass populäre gegenwartsdiagnostische Bücher durch ihre Popularität eine Themensetzung bestimmen oder zumindest die Eckdaten eines Diskurses verschieben oder vereinnahmen konnten..

Schließlich standen populäre gegenwartsdiagnostische Werke wie Gruhls Buch in einem marktlichen Kontext. Verlagsstrategien, technische Entwicklungen und innovative Verkaufsmethoden, wie etwa das von Reinhard Mohn äuBerst erfolgreich aus den Vereinigten Staaten importierte Buchclubmodell, das von den späten 1960er Jahren an dem Bertelsmann-Verlag Verkaufserfolge bescherte und dessen Veröffentlichungsstrategie sich auf einige wenige Bücher, die als Events gestaltet wurden, helfen den Erfolg von Ein Planet wird geplündert zu erklären. ${ }^{21}$

\section{Der Politiker}

Herbert Gruhl wurde 1921 in Gnaschwitz in der Oberlausitz geboren, „,nahe der schlesischen Grenze und dem Sudetenland“, wie er in einem für die Parteiwerbung in der Bundestagswahl 1969 angefertigten Lebenslauf schreibt. ${ }^{22}$ Als Bauernsohn besuchte er die Volksschule am Ort und arbeitete dann auf dem elter-

20 Siehe hierzu: Gérard Genette: Paratext. Das Buch vom Beiwerk des Buches. Frankfurt 2001. Zur Fruchtbarmachung Genettes für nicht-narrative Literatur in der geschichtlichen Betrachtung siehe: Anna Karla: Revolution als Zeitgeschichte. Memoiren der Französischen Revolution in der Restaurationszeit. Göttingen 2014. S. 116.

21 Zur Entstehung des Book-of-the-Month Club in den USA siehe Janice A. Radway: A Feeling for Books. The Book-of-the-Month Club, Literary Taste, and Middle-Class Desire. Chapel Hill u. a. 1997, S. 154-186. Zu Mohns Import des Konzepts: Richard J. Barnet und John Cavanagh: Global Dreams. Imperial Corporations and the New World Order. New York 1995, S. 70, 72 73; Richard A. Gershon: The Transnational Media Corporation. Global Messages and Free Market Competition. Abingdon 2013, S. 166; Thomas Schuler: Die Mohns. Vom Provinzbuchhändler zum Weltkonzern. Die Familie hinter Bertelsmann. Frankfurt 2004, S. 158f.

22 Herbert Gruhl: Mein Lebenslauf, in: Archiv für Christlich-Demokratische Politik (ACDP) 01699-001/2 (Bestand Herbert Gruhl). 
lichen Hof. Nach eigenem Bezeugen war er stets bildungshungrig und las während der Schulzeit vornehmlich historische Bücher. Über Gruhls Jahre als Soldat im Zweiten Weltkrieg hüllt sich der Lebenslauf in Schweigen, außer dass er ihn ,vorwiegend in Rußland und am Ende im Westen“ erlebt habe. Nach dem Krieg aus US-Kriegsgefangenschaft in Belgien geflüchtet, holte er die Hochschulreife nach und begann in Berlin mit dem Studium. Zuerst schrieb er sich an der Humboldt-Universität im Ostteil der Stadt ein, um nach der Erfahrung, dass viele seiner Lehrer in den Westen geflüchtet waren, es ihnen gleich zu tun. Nach Studium und Promotion mit einer literaturwissenschaftlichen Arbeit - wie Gruhl bemerkt, ermöglicht durch seine Frau Marianne, die er 1951 geehelicht hatte, und die während seiner letzten Studienjahre als Lehrerin den Lebensunterhalt verdiente - ging er in die freie Wirtschaft, weil er sich, wie er erklärte, vorgenommen hatte „eine möglichst große Vielseitigkeit anzustreben“. ${ }^{23}$ Er fand Arbeit bei einem Datenverarbeitungsunternehmen. Noch während seiner Berliner Zeit schloss sich Gruhl 1954 der CDU an. Er blieb der Partei auch nach seinem Umzug ins niedersächsische Barsinghausen 1959 treu. Als er dort 1961in den Gemeinderat gewählt wurde, begann seine Karriere in politischen Ämtern.

Nach Stationen als Fraktionsvorsitzender in Barsinghausen ab 1964 und Kreisvorsitzender von Hannover-Land 1965, war Gruhl ab 1969 Mitglied des Bundestags. Dort beschäftigte er sich schon früh mit Umweltfragen. Bereits 1970 verband er dieses Interesse mit einer Kritik an Wirtschaftswachstum und steigendem Konsum in einer Rede vor dem Deutschen Bundestag. Gruhl hatte nach dem zuständigen Regierungsmitglied, Innenminister Hans-Dietrich Genscher, in der zugleich ersten Bundestagsdebatte zum Thema gesprochen und dabei erklärt: „Die ökologische Betrachtungsweise muß die Folgewirkungen auf die komplexen Beziehungen aller Lebewesen in der Umwelt zur Grundlage des Kalküls machen. Wir können nicht mehr wie bisher alles unter dem ausschließlichen Gesichtspunkt des Nutzens für das wirtschaftliche Wachstum betrachten." ${ }^{24}$

Nach Gruhls Auffassung änderte sich, trotz mehrfacher dringlicher Hinweise in Reden und mitgetragener Kleiner Anfragen seinerseits, auch in den folgenden Jahren an der stiefmütterlichen, gar ,dilatorischen“ ${ }^{25}$ Behandlung

23 Ebd.; Kempf: Herbert Gruhl, S. 23-26.

24 Gruhl schoss auch in Richtung der Futurologie, dem anderen großen Lieferanten von Orientierungswissen in gegenwartsdiagnostischer Form: „Ich finde es erstaunlich, wie wenig die angeführten Zusammenhänge bei den auf das Jahr 2000 fixierten Überlegungen und Prognosen unserer Futurologen eine Rolle spielen. Die meisten ihrer optimistischen Aussagen gehören wahrscheinlich heute schon in den Papierkorb."Herbert Gruhl: Rede vor dem Deutschen Bundestag am 16. Dezember 1970, in: Deutscher Bundestag, Stenographischer Bericht. Protokoll, S. 4805

25 Ebd. 
des Umweltthemas wenig. Eine Rede, die Gruhl vier Jahre später, kurz vor der Veröffentlichung seines Buchs, im Bundestag hielt, erlaubt Rückschluss auf die Frustration, die der Politiker dadurch empfand: „Während dieses Haus solche Vorlagen wie z. B. in dieser Woche ,zur Festlegung der im Falle von Störungen auf dem Reismarkt anzuwendenden Grundregeln, oder ,zur Angleichung der Rechtsvorschriften der Mitgliedstaaten über kosmetische Mittel, vorgelegt bekommt und ausdrücklich durch Abstimmung billigen muß, sind wir mit der Frage von völlig anderer Größenordnung, wieviel Kernkraftwerke in der Bundesrepublik Deutschland gebaut werden sollen oder dürfen, überhaupt nicht befaßt. ${ }^{\text {"26 }}$

Das Dilatorische erstreckte sich dabei nicht nur darauf, mit welchen Themen der Bundestag sich befasste, sondern auch darauf, wann über ungeliebte Themen geredet werden konnte. In der Sitzung vom 22. Januar 1976 durfte Gruhl erst um 22:50 Uhr, keine halbe Stunde vor Ende der Debatte, seine Ausführungen zur Energiepolitik beginnen. Diese wurden dann nicht mehr diskutiert. Ein anwesender Korrespondent der Augsburger Allgemeinen Zeitung nahm das offensichtliche Manöver der Fraktion, Gruhl davon abzuhalten, mediale Präsenz zu erlangen, zum Anlass, einen generellen Kommentar darüber abzufassen, ,Wie die Fraktionen ihre Außenseiter behandeln“.${ }^{27}$

Schon 1970 war Gruhl Mitglied der Arbeitsgruppe Umweltfragen der CDU/CSU-Fraktion geworden. ${ }^{28} 1972$ hatte er als Vorsitzender des Unterausschusses Umweltfragen der Fraktion ein Programm für Umweltvorsorge maßgeblich verfasst. Er saß dem Gremium bis 1976 vor. ${ }^{29}$ Dessen Arbeit stand allerdings bei den anderen ihm angehörenden Fraktionsmitgliedern nicht immer ganz oben auf der Prioritätenliste. Schon 1972 hatte Gruhl in einem Einladungsbrief an die Mitglieder des Ausschusses für dessen nächsten Tagungstermin nachdrücklich um Anwesenheit bitten müssen: „Bei der letzten Sitzung waren nur vier Teilnehmer erschienen, sodaß auf die programmäßigen Beratungen verzichtet wurde. Aus diesem Grund bitte ich Sie, an der Sitzung teilzunehmen. "30

26 Herbert Gruhl: Rede vor dem Deutschen Bundestag am 14. März 1975, in: Deutscher Bundestag, Stenographischer Bericht, S. 10886.

27 Siegfried Michel: Wenn es Nacht wird in Bonn. Wie die Fraktionen ihre Außenseiter behandeln, in: Augsburger Allgemeine Zeitung, 27. Januar 2016 (ACDP 01-699-001/3).

28 Mitglieder waren u. a. der spätere Präsident des Deutschen Roten Kreuzes, Botho Prinz zu Sayn-Wittgenstein und der katholische Gewerkschaftler Günter Volmer, Vater des späteren Grünen-Politikers Ludger Volmer. Mitgliederliste CDU/CSU-Arbeitsgruppe Umweltschutz, in: ACDP 01-699-005/1.

29 Protokoll Sitzung Arbeitsgruppe Umweltfragen der CDU/CSU-Fraktion, 26. Mai 1970, in: ACDP 01-699-005/1; Kempf: Herbert Gruhl, S. 118

30 Herbert Gruhl: Brief an die Mitglieder des Unterausschusses Umweltfragen im Bundesausschuß Strukturpolitik, 06. Januar 1972 in: ACDP 01-699-005/1. 
Gruhl war seine Außenseiterposition bewusst, und er entfremdete sich mehr und mehr von der Partei. Bei der CDU-Spitze stießen seine Umwelt-Vorstöße auf wenig Gegenliebe. Die damit verbundenen Probleme, für Gruhl konservative Selbstverständlichkeit und größte, akute Aufgabe der Menschheit, tat man als Kassandrarufe ab. Helmut Kohl ignorierte das wiederholte Ersuchen Gruhls um ein Gespräch über Umweltpolitik.

Gruhl hatte den ersten Brief mit Bitte um ein Gespräch schon vor Veröffentlichung seines Buches an Kohl gesendet. Nachdem eine Antwort scheinbar ausgeblieben war, hakte er im November nach und übersandte Kohl ein Exemplar des inzwischen veröffentlichten Buches. Scheinbar war die Bitte aber wieder erfolglos. Nachdem ihm mehrmals mit Ausschluss aus der CDU gedroht worden war, machte Gruhl schließlich im Spiegel 1978 öffentlich seinem Unmut Luft und trat bald darauf selbst aus der Partei aus.. ${ }^{31}$

Er gründete 1978 zuerst die Grüne Aktion Zukunft (GAZ) und führte diese dann bei Gründung der Grünen in die neue Partei über. Politische Auseinandersetzungen mit den linken Gruppierungen, welche die Grünen mitformten einerseits, und persönliche Differenzen andererseits - Gruhl sah sich stets in einer Anführerposition und wehrte sich gegen die basisdemokratischen Anwandlungen der neuen Partei - ließen ihn 1981 schon wieder austreten. ${ }^{32}$ Die Gründung der Ökologisch Demokratischen Partei (ÖDP) 1982 sah er als Möglichkeit, konservative Umweltpolitik zu betreiben. Die ÖDP-Wahlwerbung wies dann auch darauf hin, dass ,Was die Grünen bei der SPD bewirken, das möchten wir gerne bei den sogenannten ,christlichen' Parteien." ${ }^{\text {"33 }}$ Mit der neuen Partei hatte Gruhl indes nur mäßigen Erfolg. Auch mit dieser Partei zerstritt er sich und trat 1990 aus. Gruhls Unwillen, sich klar von „ökologisch verbrämten rechtsextremen Positionen“ zu distanzieren, spielte zum Ende seiner politischen Karriere die wichtigste Rolle bei seinem unfreiwilligen Austritt aus der ÖDP. 1991

31 ACDP 01-699-001/3. „Ich lasse mir kein Ultimatum stellen“. SPIEGEL-Interview mit dem CDU-Abgeordneten Herbert Gruhl über seinen angedrohten Parteiaustritt, in: Der Spiegel, 19. Juni 1978, S. 24f. In diesem Interview äußert Gruhl den Satz „... daß es ein Wahnsinn ist, wie wir zur Zeit die Welt bewirtschaften" der diesem Artikel überschrieben steht. Vgl. auch Mende „Nicht rechts, nicht links“, S. 78. Kohl sprach nach Gruhls Tod davon, dass er das Zerwürfnis mit Gruhl bedauere, ebd.; Kempf: Herbert Gruhl, S. 16.

32 Für Gruhl war die Notwendigkeit einer gut organisierten Grünen Partei ebenso dringlich wie deren neue, experimentelle Organisationsform der Sache abträglich. Mende: „Nicht rechts, nicht links", S. 86f.

33 ACDP 01-699-011/1. Vgl. auch Daniel Hildebrand: Herbert Gruhl und die ökologische Protestbewegung, in: Historisch-Politische Mitteilungen 1 (2003), S. 325-332, hier S. 330. Zur ÖDP siehe: Uwe Kranenpohl: Die „christlichen Grünen“. Sozialstruktur und politische Einstellungen der Mitglieder der Ökologisch-Demokratischen Partei (ödp), in: Historisch-Politische Mitteilungen. 15 (2008), S. 47-62; Heiko Biehl/Uwe Kranenpohl: Große Politik in einer kleinen Partei. Strukturen und Determinanten innerparteilicher Partizipation in der Ökologisch-Demokratischen Partei (ÖDP), in: Mitteilungen des Instituts für Deutsches und Internationales Parteienrecht und Parteienforschung. 17 (2011), S. 93-109. 
schloss er sich dem rechtsextrem-ökologischen Dachverband Unabhängige Ökologen Deutschlands (UÖD) an und vollzog so endgültig den von Erhard Eppler schon 1983 konstatierten „Fall ins politische Nichts“. Gruhl konzentrierte sich vermehrt auf die Autorenrolle und brachte 1992 sein letztes Buch Himmelfahrt ins Nichts heraus.

\section{Das Buch}

Gruhls Selbstverständnis als Autor ist nicht zu trennen von seinem Selbstverständnis, ,etwas mehr Wissenschaftler als Politiker” zu sein. ${ }^{34}$ Sein Buch war allerdings, bei allen akademischen Verweisen, flüssig geschrieben und verständlich aufgebaut. Schon in seiner 542-seitigen Dissertation über Hugo von Hofmansthal hatte er es verstanden, sein Denken zugänglich zu strukturieren. Sein Einstand in die Sphäre populärer Sachliteratur war zudem in verständlicher Sprache verfasst. ${ }^{35}$

Ein Planet wird geplündert wurde $1975 \mathrm{im} \mathrm{S.} \mathrm{Fischer-Verlag} \mathrm{veröffentlicht.}$ Das Buch begründete auch die ,,alternativ“ Reihe des Verlags mit. ${ }^{36}$ Eine Bertelsmann-Buchclubausgabe folgte 1977. Eine englische Fassung existierte zwar spätestens 1976, die geplante Veröffentlichung seitens des Londoner Medienunternehmers Robert Maxwell in dessen Pergamon-Verlag erfolgte jedoch letztendlich nicht. ${ }^{37}$

Gruhl konnte für sein Werk auf mehrere literarische und intellektuelle Traditionen zurückgreifen. Ein Planet wird geplündert vereint Ziele und Stilmittel

34 „Zwischentöne: Herbert Gruhl im Interview mit Werner Krause”, Deutschlandfunk, 6. Juni 1993. Zitiert in Kempf: Herbert Gruhl, S. 219.

35 Vgl. Inhaltsverzeichnis zu Herbert Gruhl: Hugo von Hofmannsthal. Die existenziellen Grundlagen und geistesgeschichtlichen Bezüge seines Werkes. Dissertation, Freie Universität Berlin, 1956. Zitiert in Kempf: Herbert Gruhl, S. 325.

36 Gruhls Buch verschaffte der 1975 eingeführten Taschenbuchreihe gleich zu deren Beginn eine hohe Sichtbarkeit. Das Buch kam als zweites der Reihe heraus. Der Verlag selbst betrachtet es als „Grundstein der Reihe“ (http://www.fischerverlage.de/ftv/historie/html/1975_2.html, Abruf: 24. Mai 2016).

37 Brief Herbert Gruhls an Robert Maxwell betreffend Übersetzung ins Englische, in: ACDP 01699-001/3. Volker Kempf: Herbert Gruhl, S. 16, zitiert Gruhls Sohn Andreas für die Behauptung, die Veröffentlichung in Großbritannien habe auf Veranlassung wirtschaftlicher Interessen, die die Rechte für das englischsprachige Ausland aufkauften, nicht stattgefunden. Ob diese Kausalität in ihrer Einfachheit so zu halten ist, mag bezweifelt werden. Maxwell hatte erst 1974 wieder die Kontrolle über sein Medienimperium zurückerlangt und war verschiedentlich noch mit dessen Ausrichtung beschäftigt. Darüber hinaus waren Übersetzungen deutscher Sachbücher im englischsprachigen Ausland selten erfolgreich. Jedoch berührte das Buch tatsächlich direkte Wirtschaftsinteressen im Vereinigten Königreich, wie die der Erdöl- und Kohleindustrie; Richard Abel/William Gordon Graham: Immigrant Publishers. The Impact of Expatriate Publishers in Britain and America in the 20th Century. Piscataway 2009, S. 139. 
mehrerer Gattungen Sachliteratur. ${ }^{38}$ Zum einen lässt es sich als konservative Kulturkritik lesen, wie sie in den 1970er Jahren mit Besinnung auf eine frühere Tradition wiederentdeckt wurde. So versammelte Günter Rühle, Leiter der Feuilleton-Redaktion der Frankfurter Allgemeinen Zeitung, 1977 einen Kreis an Akademikern und Intellektuellen für kurze Artikel über Zeitanalysen der vergangenen Jahrzehnte. Diese Vignetten hatten das Ziel, die älteren Pubikationen auf ihre gegenwärtige Relevanz abzuklopfen und den Lesern wieder ins Gedächtnis zu rufen. In einer Publikation der Essays in Buchform unter dem Titel Bücher die das Jahrhundert bewegten. Zeitanalysen wiedergelesen sprach Rühle im Nachwort von einer „neuen Kulturkritik“ der 1970er Jahre und griff Gruhl und Ein Planet wird geplündert als einziges namentliches Beispiel heraus. ${ }^{39}$ Kulturkritik und Kulturpessimismus freilich haben ebenso Vorgänger in antiken Beschreibungen des Abfalls von einem ,goldenen Zeitalter“, religiösen Jeremiaden allgemein und deren säkularisierten Versionen seit der Aufklärung im Besonderen. ${ }^{40}$

Ein zweites Vorbild für Gruhls Buch war ein Genre des Sachbuchs, das sich seit dem 19. Jahrhundert die Popularisierung von Wissen auf die Fahnen geschrieben hatte. In der als sich beschleunigend wahrgenommenen Zeit von in die Industrialisierung übergehenden Gesellschaften hatten sich Leser in Europa und Nordamerika über die bröckelnde Gewissheit in einer vormals dominant durch Religion geprägten Umwelt durch wissenschaftlich fundierte, sich oft gar als weltliche Versionen religiöser Heilsverheißungen gerierenden und positionierenden Wissenschaft mit popularisierenden Sachbüchern hinweggeholfen. Diese meist naturwissenschaftliche Themen popularisierende Literatur hatte

38 Ich konzentriere mich hier auf Literaturgattungen, die ihre Anfänge im 19. Jahrhundert haben. In diesem war durch Zusammenwirken von technischer Innovation und steigender Alphabetisierung, bzw. im Falle der USA gar einer ersten Ausprägung der Konsumgesellschaft, der Konsum von Literatur in größerem Maße möglich geworden. Die Literatur hierzu ist äußerst umfangreich. Beispielsweise Kaspar Maase: Sphären des Wissens, Bühnen symbolischen Theaters, befreite Gebiete und die Unterwelt des Schundes. Die Massenkünste des wilhelminischen Kaiserreichs im Streit der Generationen, in: Sigrid Lange (Hg.): Raumkonstruktionen in der Moderne. Kultur - Literatur - Film. Bielefeld 2001, S. 207-226; Rudolf Schenda: Schundliteratur und Kriegsliteratur, in: Ders.: Die Lesestoffe der kleinen Leute. München 1976, S. 78104; Gideon Reuveni: Reading Germany. Literature and Consumer Culture in Germany Before 1933. New York u. a. 2006; Erich Schön: Die gegenwärtige Lesekultur in historischer Perspektive, in: Mitteilungen des Deutschen Germanistenverbandes 40 (Juni 1993) 2, S. 4-16.

39 Günter Rühle. „Nachwort. Das Gesicht des Jahrhunderts“ in: Ders. (Hg.) Bücher die das Jahrhundert bewegten. Zeitanalysen wiedergelesen. Frankfurt/Main 1980. S. 218-240, hier S. $239 f$.

40 Ähnliche Motive fanden sich etwa schon bei Hesiod, Rousseau und Nietzsche. Siehe Georg Bollenbeck: Eine Geschichte der Kulturkritik. Von Rousseau bis Günther Anders. München 2007; Theo Jung: Zeichen des Verfalls. Semantische Studien zur Entstehung der Kulturkritik im 18. und frühen 19. Jahrhundert. Göttingen 2012; Ole Petras und Kai Sina (Hg.): Kulturen der Kritik. Mediale Gegenwartsbeschreibungen zwischen Pop und Protest. Dresden 2011. 
durchaus auch schon im 19. Jahrhundert einen zeitdiagnostischen Aspekt gehabt. So kann man Justus Liebigs Chemische Briefe, einen der bekanntesten frühen Vertreter dieser Literatur im deutschsprachigen Raum, in Teilen auch als Zeitdiagnose lesen. ${ }^{41}$

Drittens knüpft Gruhls Buch an eine Tradition der soziologischen Zeitdiagnose an, die als hybrides Genre stets sowohl in die Wissenschaft als auch in die Gesellschaft hinein zu wirken suchte. ${ }^{42}$

Eine viertes Genre, in dessen Kontext Ein Planet wird geplündert stand, war das der „Self-Help“ Bücher, wie sie vornehmlich einer anglo-amerikanischen Traditionslinie aus dem 19. Jahrhundert folgend seit den 1930er Jahren in den Vereinigten Staaten populär geworden und in den 1960er Jahren in vermehrter Anzahl in den Buchmarkt gedrungen waren. ${ }^{43}$

Durch das Zusammenwirken dieser sich teils untereinander schon beeinflusst habenden Literaturarten - so griff die soziologische Zeitdiagnose stets Stränge von wissens- und wissenschaftspopularisierender Sachbuchliteratur wie auch der Kulturkritik auf - bildete sich im Übergang der 1960er auf die 1970er Jahre eine Form der populären Gegenwartsdiagnose heraus.Das Neue daran war vornehmlich, dass sich in ihr die genannten Traditionslinien trafen, und zu einem eigenständigen Genre vermischten, das als potentiell verkaufsstark angesehen und dementsprechend beworben wurde.

So stellte der S.-Fischer-Verlag Gruhls Buch im September 1975 auf einer eigens dafür einberufenen und „stark besuchten“ Pressekonferenz in Bonn vor. ${ }^{44}$ In einem ganzseitigen Bericht unter der Überschrift „Anatomie eines Bestsellers“ dekodierte der Buchreport im März 1976, wie Verlag und Autor den Erfolg von Ein Planet wird geplündert herbeigeführt hatten:

„Während das Manuskript für den Satz vorbereitet wurde, arbeiteten Werbung und Marketing an einer maßgeschneiderten Kampagne zur Einführung des Titels. [...] Pressekonferenz, Anzeigen in der Presse, Sonderdekorationen

41 Andreas Franzmann. „Der ,gebildete Laie“ als Adressat des Forschers. Sequenzielle Analyse von Titel und Vorrede zur ersten Ausgabe von Justus von Liebigs ,Chemischen Briefen“ von 1844“, in: Kretschmann: Wissenspopularisierung, S. 235-255, hier S. 241, 244, $249 f$.

42 Ute Volkmann: „Soziologische Zeitdiagnostik. Eine wissenssoziologische Ortsbestimmung“, in: Soziologie 44, S. 139-152; Uwe Schimank: „Zeitdiagnose, soziologische“, in: Werner Fuchs-Heinritz u. a. (Hg.): Lexikon zur Soziologie. 5. Auflage Wiesbaden 2013, S. 765. Zur Genreabgrenzung soziologischer Zeitdiagnosen siehe Osrecki: Diagnosegesellschaft, S. 74-85 insbes. S. 76f., 80f. Breiter zu soziologischen Zeit- und Gegenwartsdiagnosen ebd., passim; Ute Volkmann/Uwe Schimank (Hg.): Soziologische Gegenwartsdiagnosen. 2 Bde. Wiesbaden 2000-2002.

43 Sandra K. Dolby: Self-Help Books: Why Americans Keep Reading Them. Urbana u. a. 2005, S. 11; Birgitta Koch-Linde: Amerikanische Tagträume. Success und Self-Help Literatur der USA. Frankfurt/Main 1984.

44 S, Fischer Pressedienst. Januar 1977, S. 5. in: Deutsches Literaturarchiv Marbach (DLA), Sammlung der Stadt Dortmundt. Mediendokumentation zu Herbert Gruhl. 
in den Buchhandlugen und eine Sonderausgabe des Fischer-Pressedienstes, die Pressechef Uwe Gruhle über den sonstigen Empfängerkreis hinaus an ,2000 umweltengagierte Bürger' schickte, brachten das Buch ungewöhnlich schnell ins Gespräch.“ Auch Gruhl tat seinen Teil, und sprach ,,auf mehr als 50 Veranstaltungen, die sich mit Fragen des Umweltschutzes beschäftigten“. Resultat waren schon Ende Februar 197670.000 verkaufte Exemplare. ${ }^{45}$

In Ein Planet wird geplündert vereinte Gruhl akademische Belesenheit mit Anbindung an Populärkultur und Populärwissenschaft. Allein das Einführungskapitel enthält auf nicht einmal zwanzig Druckseiten Verweise auf grundlegende Denker des bildungsbürgerlichen Kanons wie Nietzsche, Hegel, oder Goethe, aber auch auf die Bibel, auf Karl Marx, Adam Smith, den Dramatiker Eugène Ionesco, den Futuristen Bertrand de Jouvenel oder die Philosophin Jeanne Hersch sowie Dennis Meadows als Vertreter des Autorenteams von Die Grenzen des Wachstums. ${ }^{46}$ Den populärwissenschaftlichen Sachbuchautor Theo Löbsack führte Gruhl ebenso als Sekundanten ins Feld wie den Physiker und Fernsehmoderator Heinz Haber und den US-Science-Fiction-Autor und kontroversen Populärphilosophen Philip Wylie. ${ }^{47}$

Die Zitatliste verdeutlicht Gruhls Anspruch, naturwissenschaftliche Erkenntnis als unleugbare positivistische Fakten darzustellen. Diese wollte er aber sowohl philosophisch-normativ in einen Wertekanon, der letztlich auf eine Tradition konservativer Kulturkritik rekurrierte, einbetten als auch populär anbinden. Die Bibliographie versammelt eine Fülle von Denkern verschiedenster Richtungen und Disziplinen, die Gruhl synthetisch in den Dienst seiner Wachstumskritik stellt. Interessant ist dabei die rhetorische Strategie, bei gewissen Autoren durchaus bedeutende philosophische Unterschiede zu seiner eigenen Analyse nicht zu erwähnen. Aus Gruhls wohlwollenden Zitaten von Philip Wylies Aufrufen zur Beschränkung und einer ökologischeren Weltsicht etwa lässt sich Wylies radikale Religions- und Christentumskritik, die dem Christlichen Demokraten Gruhl sauer aufgestoßen sein muss, nicht nachvollziehen. Dadurch schafft Gruhl einen größeren gemeinsamen Standpunkt für das im Folgenden zu analysierende ,wir“ des Buches. ${ }^{48}$

45 Anatomie eines Bestsellers: „Auch der Autor hat sich entsprechend engagiert“ in: Buchreport, Nr. 10. 5. März 1976, S. 48. DLA. Sammlung der Stadt Dortmund. Mediendokumentation zu Herbert Gruhl.

46 Der Zweiklang aus Die Grenzen des Wachstums und Ein Planet wird geplündert gehörte in der Bundesrepublik seit Erscheinen von Gruhls Buch im Umweltdiskurs zusammen. Die deutsche Übersetzung von Ugo Bardis vierzig Jahre nach dem Club of Rome-Bericht veröffentlichtem The Limits to Growth Revisited trug dann auch folgerichtig in Rückgriff auf Gruhl den Titel Der geplünderte Planet. Ugo Bardi: Der geplünderte Planet. Die Zukunft des Menschen im Zeitalter schwindender Ressourcen. München 2013.

47 Gruhl: Planet, S. 11-27.

48 Vgl. Gruhl: Planet, S. 17; Wylie: Wundertier, passim; Clifford P. Bendau: Still Worlds Collide. Philip Wylie and the End of the American Dream. San Bernardino 1980, S. 37-39. 
Die Darstellung ist eine bewusst populäre Vereinfachung der Argumente. Gruhls Gestus ist dabei jedoch gerade nicht der einer hierarchischen Autorität, wie er Wissens- und vor allem Wissenschaftspopularisierer in Deutschland in der ersten großen massenmedialen Popularisierungswelle ausgezeichnet hatte. Fern von einer simplen „Herabsetzung“ technischen Wissens und wissenschaftlicher Erkenntnis für ein ungebildetes Publikum setzte sie stets eine kommunikative Auseinandersetzung mit den Ergebnissen der Forschung voraus.

Popularisierte Wissenschaft bestimmte zugleich das Bild von Wissenschaft in der Gesellschaft maßgeblich, und erfolgreich popularisierte Disziplinen oder Teildisziplinen konnten durch Verweis auf ihre Popularität gesellschaftliche Relevanz anmelden und politischen Einfluss entfalten. In Deutschland bestimmte dabei eine hierarchisch-vermittelnde Expertenposition der Popularisierer den Grundton solcher Literatur: popularisierte Wissenschaft sollte in keiner Weise zur Partizipation von Amateuren an der von Wissenschaftlern durchgeführten Erkenntnissuche führen. Vielmehr schrieben die Vermittler, die oft selbst Wissenschaftler waren, über Wissenschaft als arkane Betätigung einer hochqualifizierten säkularisierten Hohepriesterschaft, in die Zugang zu finden nur den Auserwählten und Privilegierten vorbehalten war. In England dagegen setzte sich die Stimme des Popularisierers als „Mitnehmer“ durch. „Popularized science“" war etwas auf das der Leser freien Zugriff hatte, nachdem der Autor, der lediglich über einen Wissensvorsprung - mehr akademische Artikel gelesen zu haben, mehr Experimente selbst durchgeführt oder technische Gerätschaften selbst bedient zu haben - verfügte. Leser konnten sich in einem solchen Verständnis von Wissenspopularisierung derartige Fähigkeiten, die richtige Ausbildung und den nötigen Enthusiasmus vorausgesetzt, durchaus aneignen. ${ }^{49}$

Gruhls Buch zeugt von einem klaren Bild seines Publikums als dem ,typischen bildungsbeflissenen Sachbuchleser". ${ }^{50}$ Gut drei Generationen nach den viktorianischen und wilhelminischen Popularisierern bediente er sich nun auchdes zweiten, zugänglicheren britischen Modus der Verbreitung von Fachwissen.

Schloss Gruhls Buch in der Art der Popularisierung an Vorgänger aus dem 19. Jahrhundert an, so waren die wissenschaftlichen Erkenntnisse, die es zu Grunde legte, durchaus aktuell. Ein Planet wird geplündert ist durch und durch beeinflusst von den kybernetischen und systemtheoretischen Debatten seiner

49 Carsten Kretschmann, Einleitung: Wissenspopularisierung - ein altes neues Forschungsfeld in: Carsten Kretschmann (Hg.). Wissenspopularisierung. Konzepte der Wissensverbreitung im Wandel. Berlin 2003. S. 7-22.

50 Wie es der Journalist Harald von Troschke in einem zeitgenössischen Interview mit Konrad Lorenz treffend formulierte. Harald von Troschke: Interview mit Konrad Lorenz. Bayerischer Rundfunk, 17. Dezember 1973, in: Archiv des Bayerischen Rundfunks (BR). 
Entstehungszeit. ${ }^{51}$ Für den Geschäftsführer eines Datenverarbeitungsunternehmens scheint das nur folgerichtig. Teil I heißt denn auch „Der natürliche Regelkreis“ und diskutiert in drei Unterkapiteln „Die Natur“, „Der Mensch in der Natur“ und „Der Mensch und der Raum“, das Funktionieren des Ökosystems und den Ort des Menschen darin. Illustrierend zur Seite stehen Gruhls Prosa Schaubilder, die in Schul- und Einführungsbüchern nicht fehl am Platze wären. ${ }^{52}$

Als Auslöser für die Niederschrift des Buchs gibt er an, Die Grenzen des Wachstums rezipiert und die Notwendigkeit verspürt zu haben, dessen Computermodelle erklärend zu begründen. Die computermodellierten „Kurven“ des Club of Rome ,endeten alle sozusagen im Nichts. Er [der Club of Rome] hatte aber nicht begründet, warum das so laufen muß; er hatte nur die Kurven errechnet, warum das so ist. " ${ }^{\text {53 }}$ Gruhl strebte demnach eine Metapopularisierung und weitere Verbreitung der ohnehin schon breit rezipierten Club-of-Rome-Studie an, ohne diese grundsätzlich in Frage zu stellen.

Ein ,mission statement“ findet sich plakativ auf dem Rückumschlag: „Nicht nur der Mensch bestimmt den Fortgang der Geschichte, sondern die Grenzen dieses Planeten Erde legen alle Bedingungen fest für das, was hier noch möglich ist... Diese totale Wendung bedeutet, daß der Mensch nicht mehr von seinem Standpunkt aus handeln kann, sondern von den Grenzen unserer Erde ausgehend denken und handeln muß. "54

Wird hier noch zusammenfassend die Thematik dargestellt, so zeigt der nächste Satz, worum es Gruhl geht: „Wir nennen diese radikale Umkehr die Planetarische Wende. "55

Angefangen vom Titel mit seiner gefälligen, aber erst auf den zweiten Blick auffälligen Alliteration, die für den Umschlag gewählten Zitate, über die Widmung des Buches „Unseren Kindern“ hin zur auktorialen Form des inklusiven „wir“ im und auch schon auf dem Buch, setzten Autor und Verlag ganz auf Verständlichkeit und Zugänglichkeit für eine möglichst große Bevölkerungsschicht. Die „Wir"-Form, die Gruhl für sein Buch wählte, erlaubte es ihm zwischen sich selbst als Autor und dem Leser eine Verbindung herzustellen und zu

51 Zur Verbindung beider Ansätze: Wolfram Lutterer: Eine kybernetische Systemtheorie. Über Gregory Batesons Steps to an Ecology of Mind (1972), in: Dirk Baecker (Hg.): Schlüsselwerke der Systemtheorie. 2. Auflage Wiesbaden 2005. S. 83-91.

52 So findet sich auf S. 33 ein Abbild des ökologischen Kreislaufs von Karl Heinz Kreeb. Gruhl: Planet, S. 33.

53 Herbert Gruhl: Überleben ist Alles. Erinnerungen. München u. a. 1987, S. 154. Vgl. auch Kempf: Herbert Gruhl, S. 106.

54 Gruhl: Planet, Umschlag sowie S. 225.

55 Ebd., Umschlag sowie S. 226. 
vermitteln. Weiter schuf ein ,wir“ im Text eine produktive Ambivalenz zwischen diesen Polen. Nicht immer ist im Text klar, ob das „wir“ für Autor, Leser, beide oder für die Gesellschaft als Ganzes steht.

Ein Beispiel dieser unsicheren Position findet sich schon im Einleitungskapitel: „Wir wagen die Voraussage: wenn alles wie bisher weiterläuft, wird uns ihr Tun und Wissen [der ,Naturvölker'] bald bitter not tun, um zu überleben!“” Hier spricht Gruhl als Autor unter Verwendung des „wir“ nur für sich selbst. Der folgende Satz verdeutlicht dann aber gleich die Ambivalenz: „In der Kriegsgefangenschaft erfuhren wir hochzivilisierten Weltbürger schon einmal, was die Grundbedürfnisse eines Menschen sind: ein Mantel oder eine Decke und ein Napf für das Essen. " ${ }^{66}$ Gruhl, der selbst aus der Kriegsgefangenschaft geflohen war, kann hier zum einen aus eigener Erfahrung sprechen. Zum anderen schreibt er die Gefangenschafts- und Deprivationserfahrung einem implizit deutschen gesellschaftlichen Kollektiv zu Das „wir“ der nationalen Gesellschaft hat somit eine Kriegsgefangenen-Erfahrung gemacht. Klar gewesen sein muss Gruhl allerdings, dass im Jahr 1975 ein Großteil seiner Leser, eben die aus der Bildungsexpansion nach Wissen verlangenden Sachbuchleser, einer jüngeren Generation angehörten und auch die ebenso zu jener Zeit expandierende Zahl an weiblichen Leserinnen diese spezifische Erfahrung nicht direkt teilen konnte. „Wir“ steht hier demnach gleichzeitig für drei Ebenen: für den Autor, den Leser, und die Gesellschaft.

Auch anderswo ist Gruhls Duktus neben dem des aufrüttelnden Bußpredigers stets einer des Mitnehmens und Verständlichmachens. So beginnt er Teil I seines Buches mit der rhetorischen Frage: „Wodurch entstand das Leben auf unserem Planeten?“ Antwort: „Durch Energieumwandlung. Alle Energie wird zunächst von der Sonne geliefert. Diese war und bleibt die Grundvoraussetzung des Lebens auf unserem Planeten. " ${ }^{\text {" }} 7$ Ähnlich ging der Autor einer weiteren höchst einflussreichen Gegenwartsdiagnose vor. Alvin Toffler fragte in $\mathrm{Der} \mathrm{Zu}$ kunftsschock: „Aber wie wissen wir überhaupt, daß der Wandel sich beschleunigt? [...] Um sehr unterschiedliche Prozesse miteinander vergleichen zu können, bedürfen wir eines Maßstabs. Dieser Maßstab ist die Zeit. “58 Im Gegensatz zu Tofflers impressionistischem Herunterbrechen von Komplexität auf poppige Metaphern von Barbiepuppen und papiernen Hochzeitskleidern als Menetekel der Wegwerfgesellschaft ist Gruhl gewissermaßen bodenständiger. ${ }^{59}$ Auf effekthaschende Zwischenüberschriftenverzichtet jedoch auch er nicht. Findet man bei Toffler eine „Wanderung in die Zukunft“, „Die Freudsche Welle“ oder „Informationsüberfütterung“, so bietet Gruhl „Der Wald verbrennt - die Wüste

56 Ebd., S. 13

57 Gruhl: Planet, S. 31

58 Alvin Toffler: Der Zukunftsschock. Stuttgart o. J., S. 21

59 Ebd., S. 43-45, S. 397-400; Alvin Toffler: Future Shock. New York 1970, S. 52. 
wächst“, „Der Zahlenkult“ und „Die Raumschiff-Wirtschaft““ ${ }^{60}$ Auch verlangt er seinem Publikum durch wiederholte Verweise auf zwar erläuterte, aber keineswegs einfache mathematische Formeln mehr Denkarbeit ab. Stilistisch - im Sinne von klarer Sprache, Verwendung von sprechenden Analogien unter weitgehender Vermeidung von Fachjargon - ist Gruhl auch Konrad Lorenz vergleichbar, dessen biologistischer Kulturkritik er in einigen Kapiteln durchaus nahe steht. ${ }^{61}$

Wichtig ist hierbei nicht die Frage ob es eine mögliche Kausalität zwischen Gruhls Rezeption von Büchern wie Der Zukunftsschock und einer Emulation des Stils seinerseits gegeben hat. Entscheidend ist, dass Gruhls Buch sich eines Stils bediente, der sich ähnlich in anderen populären Gegenwartsdiagnosen wiederfand. Damit konnten Leser das Werk einem Genre leicht verständlicher Literatur zurordnen, derenInhalte als wissenschaftlich und neutral angesehen wurden. Mag es auf den ersten Blick erstaunen, dass das Buch eines konservativen CDU-Abgeordneten auch von K-Gruppen-Mitgliedern rezipiert wurde und werden konnte, so ist die zwar unmissverständlich angelegte, aber nie explizit eingeforderte Verbindung von wissenschaftlicher Erkenntnis und Wertvorstellungen mit ein Grund dafür.

Während Teil I mit kaum zwanzig Seiten knapp ausfällt, bilden Teil II und III den Großteil des Buches. Gruhl setzt sich hier zur Aufgabe, die anfangs vorgestellte und im Titel schon vorgeschickte These akribisch zu unterfüttern. Beispiele aus diesen Buchteilen sollen illustrieren, wie er dabei verfährt.

In Teil II, „Der künstliche Produktionskreis“, geht Gruhl zu einer klaren Wachstumskritik auf Basis der Ergebnisse der Club of Rome Studie über. Wachstums- und Kulturkritik verbindet er jedoch stets mit einem Rest Glauben an die technische und gesellschaftliche Machbarkeit von Lösungen. So begegnet Gruhl dem übergeordneten Entropieproblem, das er in der Verschwendungsgesellschaft verkörpertsieht, mit einem Aufruf zur Wiederverwertung von Rohstoffen - schon mit dem Anglizismus „Recycling“ überschrieben. Gruhls fünf „Möglichkeiten zur Förderung der Wiederverwertung“ sind: 1 . Subventionierung der Wiederverwendung; 2. Verteuerung der originären Rohstoffe; 3. Erhöhung der Nutzungsdauer der Produkte; 4. Produktgestaltung im

60 Gruhl: Planet, S. 7-9.

61 Vor allem im Kapitel II.5. Selbstausrottung durch Geburten? Auf S. 170-182. Hier fallen Sätze wie „Die heutige angeblich vernunftbegabte Menschheit verhält sich, was ihre eigene Zukunft betrifft, genauso irrational wie irgendeine Population des Tierreiches. Ja, im Tierreich reagieren wenigstens einige Arten mit dem Instinkt. Der Mensch aber hat seinen Instinkt verloren [...]. “S. 177. Vgl. Konrad Lorenz: Die acht Todsünden der zivilisierten Menschheit. München 1973, S. 107. 
Hinblick auf die Wiederverwendung und 5. Unterstützung der Forschung. Wieder übernimmt er Zahlen und Thesen aus der Club of Rome Studie, und beruft sich stellvertretend für diese auf Dennis Meadows. ${ }^{62}$

Die Verknüpfung mit impliziten Handlungsanweisungen unterscheidet unterscheidetGruhls Buch von anderen gegenwartsdiagnostischen Werken,und rückt es eher in die Nähe der Self-Help-Literatur (berühmt-berüchtigt für ihre „Zwölf Schritte“-Programme zur Besserung der persönlichen Lage). Gruhl schließt hier an eine seit den 1970er Jahren verbreitete Form der Selbsthilfeliteratur an. Diese stellt, im Gegensatz zu vorgegangenen Werken, die Individuen Hilfestellung zur Anpassung an eine sich verändernde Gesellschaft bieten wollten, die produktive Abweichung von der gesellschaftlichen Norm als kreatives Moment in den Vordergrund. ${ }^{63}$ Damit kann das Buch als gemeinsame Referenzgrundlage für nachgelagerte, oder durch es zumindest mitgeprägte soziale Gruppenaktivität, wie sie etwa in den Neuen Sozialen Bewegungen und den aus ihnen erwachsenden Parteien und Organisationen sichtbar ist, dienen. ${ }^{64}$

Teil III mit der Überschrift „,Die planetarische Wende“ gibt sich dann durchweg millenarisch. Hier zeigt sich am klarsten Gruhls kulturkritischer Ansatz. Nichts Geringeres als eine durch die Umstände erzwungene Wende des gesamten Planeten könne die eigentlich schon verspielte Zukunft noch retten. Gruhl zieht hier die „Raumschiff Erde“-Metapher, die in den 1970ern große Verbrei-

62 Interessant ist dabei, dass sich Punkt 3. und 4. fast wörtlich mit These 7 (Gutes Design ist langlebig) und 9 (Gutes Design ist umweltfreundlich) der in den frühen 1980er Jahren vom einflussreichen Industriedesigner Dieter Rams zusammengetragenen „Zehn Thesen für gutes Design“ decken. Vgl. Deutscher Designer Club (DDC): Gute Gestaltung/Good Design 11. Berlin 2011, S. 13. Eine tiefere Ergründung dieses Zusammenhangs kann an dieser Stelle nicht geleistet werden, jedoch scheint es bei der schieren Verbreitung von Gruhls Buch durchaus wahrscheinlich, dass Teile davon derart in verschiedenste gesellschaftliche Kontexte diffundierten.

63 Boris Traue: Coaching. Die Mobilisierung der Psyche, 1775-1975, in: Sabine Maasen u. a. (Hg.): Das beratene Selbst. Zur Genealogie der Therapeutisierung in den ,langen“ Siebzigern. Bielefeld 2014. S. 243-262, hier S. 247; Dolby: Self-Help Books, S. 35-55; allgemeiner: Steve Starker: Oracle at the Supermarket. The American Preoccupation With Self-Help Books. New Brunswick u. a. 1989.

64 Ich schließe hier an Thomasina Jo Borkmans Konzept der „Commons“ an, das sie für Selbsthilfegruppen und ähnliche Organisationen entwickelt hat. Commons können nach Borkman informelle Selbsthilfegruppen ebenso einschließen wie soziale Bewegungen oder politische Parteien. Sie verfügen idealtypisch über 5 Merkmale - Freiwilligkeit; ein gemeinsames Ziel; gemeinschaftliche Verfügbarkeit von Ressourcen, Techniken, Aktivitäten oder Fähigkeiten; Gemeinschaftssinn; Fairness im sozialen Umgang. Gruhls Buch fungiert hier im Sinne von Borkmans drittem Merkmal des ,idealtypischen Commons“. Es wird als gemeinschaftliche Ressource genutzt. Thomasina Jo Borkman: Understanding Self-Help/Mutual Aid. Experiential Learning in the Commons. New Brunswick 1999, S. 45-47. 
tung erlangte, als Sinnbild heran. In nur leicht anderer Form war diese allerdings als „Arche“ schon bei Ludwig Klages geschwommen. ${ }^{65}$ Die Parallele zwischen den beiden Autoren blieb zeitgenössisch nicht unbemerkt. So schrieb der Spiegel in einer Sammelrezension: „Zwar zählt sich auch Herbert Gruhl zu den ,nüchternen Realisten ‘. Aber in seinen Tiraden gegen den , arroganten Menschen", gegen eine ,Welt, in der nicht mehr der Geist, sondern die Materie herrscht", entpuppt er sich als recycleter Klages, als Second-hand-Spengler $[\ldots]^{\text {*. } 66}$

Trotz allen „Feuerspeien[s]“67 beharrt Gruhl im Schlusskapitel darauf, dass er bei der „Analyse der naturwissenschaftlichen, wirtschaftlichen und politischen Lage der heutigen Welt [...] ohne ethische Gesichtspunkte aus[gekommen sei]“. Jedoch „der Weg, der aus dem Dilemma herausführen könnte, ist dagegen ohne höhere Werte nicht zu finden“. ${ }^{68}$ Das legt die Vermutung nahe, dass Gruhl sein Buch in neutrale Analyse und dann, darauf aufbauend aber davon abgetrennt, normative Handlungsanweisungen zweigeteilt sah.

Allein: der gesamte Tenor des Werkes macht es unmöglich, das Eine vom Anderen zu trennen. Gruhls Darlegung vorgeblich neutraler Wissenschaft trägt eine Kulturkritik immer schon in sich.

\section{Die Wirkung}

Die vergleichsweise große Bedeutung und Wirkmacht grüner Bewegungen und der aus ihnen entstehenden Partei Die Grünen in Westdeutschland im Vergleich zu anderen westlichen Staaten, wie etwa Frankreich, Großbritannien oder den USA, deren Gesellschafts- und Wirtschaftsordnung in den 1970er Jahren ähnliche Wandlungen durchmachten, hat mannigfaltige Gründe. Die Sozial-, Struktur- und Ideengeschichte hat diese verschiedentlich erläutert und plausibel gemacht. Der Einfluss von Gegenwartsdiagnosen, und vor allen anderen von Ein Planet wird geplündert, wird dabei auch meist erwähnt und mit einem Seitenblick auf Gruhls Rolle als Mitinitiator der erfolgreichsten Neugründung in der Parteienlandschaft der Bundesrepublik berücksichtigt.

Gruhl war es gelungen, in einer sich selbst als krisenhaft wahrnehmenden Zeit, ein griffiges, lesbares Vademekum anzubieten. Sein Buch wurde mit schon nach einem Jahr über 100.000 verkauften Exemplaren zum Bestseller und schuf sowohl im bundesrepublikanischen Diskurs eine gegenwartsdiagnostische Formel, die die Dringlichkeit der Bekämpfung von Umweltzerstörung auf den Punkt brachte, als auch innerhalb der Neuen Sozialen Bewegungen und

65 Ebd., S. 227; Ludwig Klages: Mensch und Erde. Ein Denkanstoß. Bonn 1980, S. 17. Vgl. auch Wüst: Konservatismus und Ökologiebewegung, S. 51f.

66 Wilhelm Bittdorf: „Tut Buße! Das Ende ist nah!“, in: Der Spiegel, 23. August 1976, S. 126.

67 Ebd.

68 Gruhl: Planet, S. 341. 
der sich formierenden Grünen Partei eine gemeinsame Gesprächsgrundlage. Damit steht es exklusiv unter den gegenwarts- und zeitdiagnostischen Büchern jener Zeit. Andere Werke schafften es zwar alle, bestimmte Aspekte von Gesellschaft und Welt in den Blick zu nehmen, zu diskutieren und diskursiv anschlussfähig zu machen. Sie konnten aber kaum je in dem Maße wie Ein Planet wird geplündert direkten Einfluss sowohl auf politische Entscheidungen wie auch alltägliche Handlungen und verinnerlichte Überzeugungen einer derart bemerkenswert großen Anzahl von Bürgerinnen und Bürgern jeglicher politischer Couleur und verschiedenster Vorprägungen nehmen.

Der Einfluss des Buches resultierte jedoch nicht in einem enstprechenden Einfluss des Autors. Die Ironie dieses Bucherfolgs bei gleichzeitig stetig schwindendem direktem politischem Einfluss Gruhls hatte Erhard Eppler schon 1983 bemerkt. Er hielt fest, Gruhl habe es nicht geschafft, seinen Naturkonservatismus von einem ideologischen Konservatismus zu trennen, der inhärent das politische System zu befestigen suchte. Gleichzeitig habe er durch Überbetonung des ersteren keine politische Heimat mehr in einer Partei gefunden, die in den 1970er und 1980er Jahren diesem weitaus weniger verpflichtet war als dem letzteren. Epplers Fazit zeugt von einer schon fast griechisch-tragisch anmutenden Hamartia des Protagonisten: ,Wer die Mächtigen halbherzig, ja widerwillig ärgert und gleichzeitig allem mißtraut, was sich an der Basis bewegt, fällt ins politische Nichts. Das ist nun zwar kein Naturgesetz, dafür eine simple politische Faustregel.“69

Herbert Gruhl hatte die Zeichen der Zeit in den 1970er Jahren erkannt. Er trug maßgeblichzu Umwälzungen im politisch-gesellschaftlichen Diskurs in der Bundesrepublik bei, und sowohl direkt als auch indirekt zu deren realer Verfestigung in der Partei der Grünen. Dass er andere Zeitzeichen nicht anerkennen wollte und seinen persönlichen Geltungsanspruch als auch eine politische Überzeugung, die keiner politischen Partei zugeordnet werden konnte, nicht hintanzustellen vermochte, ließ ihn durch drei Parteien mäandern und am jeweiligen Punkt, an dem es keine Verständigung mehr untereinander gab, austreten.

$\mathrm{Zu}$ Ende seines Lebens konzentrierte sich Gruhl auf die Schriftstellerei und verfasste mit dem Spätwerk Himmelfahrt ins Nichts 1992 einen Kompagnon zu seinem zwar drängenden, aber noch Hoffnung auf Schadensbegrenzung lassenden Bestseller. Für die Umkehr, so Gruhl in diesem, noch weitaus endzeitlicher daherkommenden Buch, sei es nun schon zu spät. Die Menschheit steuere auf die Katastrophe zu und mache nicht einmal Anstalten, die Rettungskräfte schon vorzeitig zu alarmieren - ein Bild, das Gruhl noch in der Einleitung zu Ein

69 Eppler: Vom Fall in das politische Nichts, S. 88. 
Planet wird geplündert bemüht hatte. ${ }^{70}$ Im Rückblick nehmen sich Gruhls eigene Worte aus dem Nachwort zu seinem 1975er-Erfolg zumindest für die eigene Rolle prophetisch aus: „Jahrzehntausende ungeschriebener Menschheitsgeschichte und 5000 Jahre überlieferter Geschichte gebieten uns einen tapferen Versuch - auch dann, wenn wir scheitern sollten. “71

Gruhls „tapferer Versuch“, die bundesdeutsche Politik zu zwingen, dem Umweltthema Gehör zu schenken, war nicht nur, aber doch entscheidend mitbestimmt von seinen Formulierungen und seiner popularisierten Analyse, von Erfolg gekrönt. Sein eigener Erfolg als Politiker einer daraus resultierenden Bewegung blieb indes bescheiden. Gruhl war aus der konservativen Mitte der bundesrepublikanischen Gesellschaft über ein Zwischenspiel „vorne“ mehr und mehr an deren rechten Rand gerückt. Aus dem unerhörten war ein ungehöriger und schließlich ein unzugehöriger Mahner geworden.

70 Gruhl: Planet, S. 26

71 Ebd., S. 350 . 